\title{
Firm heterogeneity and total factor productivity: New panel-data evidence from Vietnamese manufacturing firms
}

\author{
Quang Thanh Ngo ${ }^{a^{*}}$ and Quang Van Trana
}

${ }^{a}$ University of Economics and Law, Ho Chi Minh National University, Ho Chi Minh City, Vietnam

\begin{tabular}{l}
\hline C H R O N I C L E \\
\hline Article history: \\
Received: November 4, 2019 \\
Received in revised format: No- \\
vember 292019 \\
Accepted: December 12, 2019 \\
Available online: \\
December 15, 2019 \\
\hline Keywords: \\
Total factor productivity growth \\
TFP distance \\
TFP dispersion \\
FDI-driven TFP \\
Spillover effects \\
Heterogeneity \\
Manufacturing firms \\
Vietnam
\end{tabular}

\section{Introduction}

The characteristics of a firm affect its productivity. For example, large firms have an advantage over small firms since they can use market power and economies of scale. Furthermore, firms' learning processes vary greatly depending on the quality of managers and workers, so learning is basically firm-specific (Bloom et al., 2016). Thus, heterogeneity in firm-specific factors determines firm heterogeneity in productivity. The new international trade theory based on firm heterogeneity determines the differences in productivity underscore (Melitz, 2003). Productivity self-selects firms participating in international trade, and exporters are more productive than non-exporters, even before entering international markets. The interaction between firm productivity and trade has been tested empirically, supporting the positive relationship between exports and productivity with overwhelming consistency (e.g. Bernard et al., 2012; Eaton et al., 2011; Kasahara \& Lapham, 2013). In investigating firm heterogeneity in trade, firms are grouped into three categories by further dividing firms engaged in trade into two groups: export (and import) and FDI. In this regard, the study also sheds light on firm heterogeneity in the productivity of manufacturing firms engaged in international trade. A recent study of Nguyen (2017) in Vietnam examined TFP changes during business reforms in Vietnam in 2000-2010 and found that technology gap in terms of TFP differs between the least productive firms and the frontier firms and the differences are narrowed down across industries (and economic regions). Most

* Corresponding author

E-mail address: thanhnq@uel.edu.vn (Q.T. Ngo)

C 2020 by the authors; licensee Growing Science, Canada doi: $10.5267 /$ j.msl.2019.12.016 
recent study by Nguyen et al. (2020) focused on labor heterogeneity in TFP in Vietnam and found that in the private sector, labor heterogeneity on productivity was very dependent on specific manufacturing sectors, and on type of TFP. This paper contributes to the empirical literature in some aspects. First, for the first time, we follow a more comprehensive approach to examine the productivity heterogeneity in Vietnamese manufacturing industry. Second, while most of the firm-level previous studies examine the productivity at aggregate level of the manufacturing sector, this study, by exploring the enriched dataset, tries to uncover the disaggregate level effects of twenty-two manufacturing sub-sectors. The study is differentiated from Nguyen (2017) and Nguyen et al. (2020) in some aspects: (1) we focus on both input and trade related heterogeneity in TFP, and (2) as mentioned above, we analyse TFP at disaggregated level of twenty-two manufacturing sub-sectors.

The paper is structured as follows. Section 2 is about the study design, in which data and TFP estimation are presented. Firm heterogeneity is also classified. Section 3 presents the analysis results. Conclusions and implications are in Section 4.

\section{Study design}

\subsection{Data}

The main data source for TFP estimation is the Vietnam Annual Enterprise Survey (VAES) which is conducted annually by the General Statistical Office (GSO) of Vietnam. The VAES provides annual data for financial performance and financial position by different industry groups. The surveys collected information on firms' activities, firm characteristics, location, industries, labor and wages, assets and liabilities, export and import of goods, and business results (including turnover, cost of goods, administration costs, net profit) at the firm level. All types of manufacturing sectors were covered in the sample. More information on the survey can be found in Ngo and Nguyen (2019).

\subsection{TFP estimation}

\subsubsection{Approach to estimate TFP}

To accomplish the objectives of this study, the proposed empirical analysis starts with the estimation of TFP at the firm level. This is accomplished separately for all twenty-two sample industries. It is noteworthy that the use of ordinary least squares (OLS) in the estimation of the production function may lead to some serious problems. As pointed out by Griliches and Mairesse (1995), profit-maximizing firms immediately adjust their inputs (in particular capital) each time they observe a productivity shock, which ensures input levels are correlated with the same shocks. Since productivity shocks are unobserved, they enter in the error term of the regression. Hence, inputs may turn out to be correlated with the error term of the regression, and thus OLS estimates of production functions are biased. Olley and Pakes (1996) (OP, hereafter) and Levinsohn and Petrin (2003) (LP, hereafter) developed two similar semi-parametric estimation procedures to overcome this problem. As claimed by Ackerberg et al. (2006) (AFC), multicollinearity could happen when labor is correlated with the proxy, then the labor coefficient cannot be identified. To overcome this issue, Wooldridge (2009) and later Petrin and Levinsohn (2012) (LP) suggested applying instruments (IVs) estimator using the own lags of labor for its instruments. Ackerberg et al. (2006) suggested a method that builds upon the ideas in OP and LP, e.g. using investment or intermediate inputs to "proxy" for productivity shocks, but does not suffer from the above collinearity problems. AFC procedure, however, unlike the OP and LP procedures, which estimate the labor coefficient in the first stage (where the collinearity issue arises), involves estimating the labor coefficient in the second stage.

In this study, we prefer the AFC methodology, which is an extension of the LP technique for the computation of TFP. This methodology explicitly recognizes and overcomes the endogeneity, which occurs because at least a part of the TFP is observed by the profit-maximizing firms early enough to allow the factor input decisions to be changed, and possible collinearity between labor and proxy variable. Specifically, we follow the value-added method of the AFC procedure and the deflated gross value added (LY) of firms is used as a measure of output. Further, in this process intermediate inputs (raw material) are used as a proxy, to avoid the bias problem.

\subsubsection{TFP measurements}

TFP growth

Eq. (1) presents how $\ln ($ TFP) can be calculated from parameters estimated from the IVs estimator by Wooldridge (2009) and Petrin and Levinsohn (2012).

$$
\widehat{\omega_{l j t}}=\widehat{v a_{\imath \jmath t}}-\widehat{\beta}_{l} l_{i j t}-\widehat{\beta_{k}} k_{i j t}
$$

where:

$\widehat{\omega}_{i j t}$ is the $\log$ of estimated TFP of firm $i$ in industry $j$ at year $t$,

$\widehat{v a_{\imath \jmath t}}$ is the value-added estimated from the production function and

$l_{i j t}, k_{i j t}$ is $\log$ values of the number of labors and real accumulated capital stocks, respectively. 
Here $\widehat{\beta_{l}}$ labor parameter, $\widehat{\beta_{k}}$ capital parameter are estimated from the production function of industry $j$.

The TFP growth rate at the firm level is calculated by the TFP difference between two points of time: $t$ and $t+1$.

\section{TFP distance}

Technological distance (technological difference): The gap in TFP is measured as the difference in TFP between a firm and the firm with the highest TFP and between a firm and the top-3 firms with high TFP.

$$
\begin{aligned}
& \operatorname{TFPgap}_{1}=\ln (\text { TFP })_{\mathrm{top} 1}-\ln (\text { TFP }) \\
& \text { TFPgap }_{3}=\ln (\text { TFP })_{\text {meanptop3 }}-\ln (\text { TFP })
\end{aligned}
$$

where:

$\ln (T F P)_{\text {top } 1}: \ln (\mathrm{TFP})$ of the firm with highest TFP

$\ln (T F P)_{\text {meantop3 }}$ : Mean of $\ln (\mathrm{TFP})$ of top-3 firms

$\ln (T F P): \ln (\mathrm{TFP})$ of a firm in consideration

As TFP is expressed in log values, the gap has an interesting implication. It can be inverted to the ratio between the output produced by the TFP frontier and the output produced by the least productive, assuming that both use the same inputs (Syverson, 2011): Ratio $=\mathrm{e}^{\mathrm{TFPgap}}$.

\section{TFP dispersion}

Technological diffusion (technological dispersion) is measured as the difference in TFP between a firm and the sector as a whole.

TFPdiff $_{l}=\ln ($ TFP $)-\ln (\text { TFP })_{\text {mean }}$,

where:

$\ln (T F P)_{\text {mean }}$ : Mean of $\ln (\mathrm{TFP})$ of the manufacturing sub-sector,

$\ln (T F P): \ln (\mathrm{TFP})$ of a firm.

\subsubsection{FDI-driven TFP spillover effects}

FDI spillover effects are measured as the difference between TFP of FDI firms and the other domestic firms in the sub-sector.

TFPspillovers $=\ln (\text { TFP })_{\text {meanFDI }}-\ln (T F P)$,

where:

$\ln (T F P)_{\text {spillovers }}$ : FDI spillover effects of TFP,

$\ln (T F P)_{\text {meanFDI }}:$ Mean of $\ln (\mathrm{TFP})$ of FDI firms, $\ln (T F P)_{\mathrm{dom}}: \ln (\mathrm{TFP})$ of domestic firms.

\subsubsection{On firm heterogeneity}

For firm heterogeneity analysis, this study considers not only firms' structural features such as size in terms of labor, capital, average wage rate, but also its business characteristics such as trade (export and import) and FDI. Although many characteristics are included to represent firm heterogeneity, special attention is paid to firms' trade features. Among the structural feature, firms with more than 5000 employees are classified as extreme-large firms, those with 1000-5000 employees are considered as upper-large firms, those with 500-1000 employees are nominated as lower-large firms, those with 300-500 employees represent the big firms, those with 200-300 employees are upper-medium firms, those with 50-200 employees are lower-medium firms and finally, those with 10-50 employees represent the small firms. The average wage rate is used as a proxy to represent human capital, which is compensated accordingly by wages. Thus, the variable is used to represent the overall quality of employees working for a firm. In the analysis, average wages are classified into four quartile ones and thus firms are compared among those belonging to each $25 \%$. Four export and FDI-related categories (domestic non-exports, FDI non-exports, domestic exports, and FDI exports) are constructed to analyze the different effects of export and FDI on productivity. Similarly, four import and FDI-related categories (domestic non-imports, FDI non-imports, domestic imports, and FDI imports) are constructed to clarify the different effects of import and FDI on productivity. 


\section{The results}

\subsection{TFP estimation}

\subsubsection{Empirical results of TFP estimation}

TFP is estimated with the Ackerberg et al.'s (2015) method, using value-added production. To accomplish the objectives of this study, the proposed empirical analysis starts with the estimation of TFP. This is performed separately for all twenty-two manufacturing sectors. Our value-added specifications include two primary inputs as regressors: laborers, and capital. The study posits a Cobb-Douglas production function and estimates production functions separately for each two-digit industry code. The estimated production function is reported in Table 1 for each sub-industry, which suggests that workers (logarithm of labor) and capital (logarithm of capital) are significant in all industries at 1 percent level of significance.

Table 1

Cobb-Douglas production function estimation using D. A. Ackerberg et al. (2015) estimator (Dependent variable: Value-added value (logarithm), 2010-2016)

\begin{tabular}{|c|c|c|c|c|c|c|c|c|c|c|c|}
\hline VARIABLES & $\begin{array}{c}\text { (1) } \\
\text { sector } 10\end{array}$ & $\begin{array}{c}(2) \\
\text { sector } 11\end{array}$ & $\begin{array}{c}\text { (3) } \\
\text { sector } 13\end{array}$ & $\begin{array}{c}(4) \\
\text { sector } 14\end{array}$ & $\begin{array}{c}(5) \\
\text { sector } 15\end{array}$ & $\begin{array}{c}(6) \\
\text { sector } 16\end{array}$ & $\begin{array}{c}(7) \\
\text { sector } 17\end{array}$ & $\begin{array}{c}(8) \\
\text { sector } 18\end{array}$ & $\begin{array}{c}(9) \\
\text { sector } 20\end{array}$ & $\begin{array}{c}(10) \\
\text { sector } 21\end{array}$ & $\begin{array}{c}(11) \\
\text { sector } 22\end{array}$ \\
\hline Capital (log) & $0.517 * * *$ & $0.800 * * *$ & $0.473 * * *$ & 0.0341 & $0.139 * * *$ & $0.389 * * *$ & $0.326 * * *$ & $0.260 * * *$ & $0.621 * * *$ & 0.162 & $0.426^{* * *}$ \\
\hline & $(0.0513)$ & $(0.145)$ & $(0.0192)$ & $(0.0227)$ & $(0.0362)$ & $(0.0349)$ & $(0.0493)$ & $(0.0596)$ & $(0.0328)$ & $(0.209)$ & $(0.0448)$ \\
\hline Labor $(\log )$ & $\begin{array}{l}0.712 * * * \\
(0.0724)\end{array}$ & $\begin{array}{l}0.605^{*} \\
(0.314)\end{array}$ & $\begin{array}{c}0.755^{* * *} \\
(0.0332)\end{array}$ & $\begin{array}{l}1.105^{* * *} \\
(0.0378)\end{array}$ & $\begin{array}{l}0.931 * * * \\
(0.0385)\end{array}$ & $\begin{array}{l}0.901 * * * \\
(0.0862)\end{array}$ & $\begin{array}{c}1.041^{* * *} \\
(0.109)\end{array}$ & $\begin{array}{c}1.117 * * * \\
(0.179)\end{array}$ & $\begin{array}{c}0.597 * * * \\
(0.0577)\end{array}$ & $\begin{array}{c}1.372 * * * \\
(0.368)\end{array}$ & $\begin{array}{l}0.789^{* * *} \\
(0.0562)\end{array}$ \\
\hline Observations & 4,865 & 485 & 2,010 & 4,445 & 1,525 & 1,820 & 1,770 & 970 & 1,825 & 525 & 2,765 \\
\hline $\begin{array}{l}\text { Wald test statistic of con- } \\
\text { stant returns to scale }\end{array}$ & 85.48 & 5.162 & 152.7 & 50.19 & 24.73 & 23.71 & 30.35 & 8.442 & 51.99 & 7.319 & 107.8 \\
\hline Sargan-Hansen test statistic & $4.19 \mathrm{e}-09$ & $3.87 \mathrm{e}-08$ & $6.84 \mathrm{e}-09$ & $3.03 \mathrm{e}-08$ & $2.89 \mathrm{e}-08$ & $1.45 \mathrm{e}-09$ & $1.78 \mathrm{e}-07$ & $4.93 \mathrm{e}-08$ & $9.18 \mathrm{e}-09$ & 0.0160 & $6.12 \mathrm{e}-09$ \\
\hline \multicolumn{12}{|l|}{ Continued } \\
\hline VARIABLES & $\begin{array}{c}(12) \\
\text { sector } 23\end{array}$ & $\begin{array}{c}(13) \\
\text { sector } 24\end{array}$ & $\begin{array}{c}(14) \\
\text { sector } 25\end{array}$ & $\begin{array}{c}(15) \\
\text { sector } 26\end{array}$ & $\begin{array}{c}(16) \\
\text { sector } 27\end{array}$ & $\begin{array}{c}(17) \\
\text { sector } 28\end{array}$ & $\begin{array}{c}(18) \\
\text { sector } 29\end{array}$ & $\begin{array}{c}(19) \\
\text { sector } 30\end{array}$ & $\begin{array}{c}(20) \\
\text { sector } 31\end{array}$ & $\begin{array}{c}(21) \\
\text { sector } 33\end{array}$ & $\begin{array}{c}(22) \\
\text { sector } 34\end{array}$ \\
\hline Capital (log) & $\begin{array}{l}0.363^{* * *} \\
(0.0659)\end{array}$ & $\begin{array}{l}0.476^{* * *} \\
(0.0138)\end{array}$ & $\begin{array}{l}0.482^{* * *} \\
(0.0429)\end{array}$ & $\begin{array}{l}0.383^{* * *} * \\
(0.0528)\end{array}$ & $\begin{array}{l}0.576^{* * * *} \\
(0.0478)\end{array}$ & $\begin{array}{l}0.472 * * * \\
(0.0662)\end{array}$ & $\begin{array}{l}0.808^{* * *} \\
(0.279)\end{array}$ & $\begin{array}{c}0.326 \\
(0.203)\end{array}$ & $\begin{array}{l}0.223 * * * \\
(0.0680)\end{array}$ & $\begin{array}{l}0.708 * * * \\
(0.102)\end{array}$ & $\begin{array}{l}0.228^{* * *} \\
(0.0488)\end{array}$ \\
\hline Labor (log) & $\begin{array}{c}0.986 * * * \\
(0.111)\end{array}$ & $\begin{array}{l}0.792 * * * \\
(0.0215)\end{array}$ & $\begin{array}{c}0.490 * * * \\
(0.108)\end{array}$ & $\begin{array}{l}0.731 * * * \\
(0.0963)\end{array}$ & $\begin{array}{l}0.620 * * * \\
(0.0660)\end{array}$ & $\begin{array}{c}0.707 * * * \\
(0.206)\end{array}$ & $\begin{array}{c}0.649 \\
(0.468)\end{array}$ & $\begin{array}{l}1.078 * * \\
(0.432)\end{array}$ & $\begin{array}{c}0.947 * * * \\
(0.106)\end{array}$ & $\begin{array}{c}0.360 * * * \\
(0.121)\end{array}$ & $\begin{array}{c}0.925 * * * \\
(0.103)\end{array}$ \\
\hline Observations & 3,770 & 620 & 3,360 & 740 & 1,040 & 810 & 440 & 570 & 2,445 & 145 & 930 \\
\hline $\begin{array}{l}\text { Wald test statistic of con- } \\
\text { stant returns to scale }\end{array}$ & 52.50 & 219.6 & 0.0583 & 3.370 & 57.84 & 1.351 & 4.571 & 3.010 & 5.093 & 0.654 & 5.511 \\
\hline Sargan-Hansen test statistic & $1.82 \mathrm{e}-08$ & $7.00 \mathrm{e}-09$ & 6.456 & $3.50 \mathrm{e}-08$ & $1.59 \mathrm{e}-08$ & $2.28 \mathrm{e}-08$ & 0.0780 & $1.65 \mathrm{e}-08$ & $8.96 \mathrm{e}-08$ & $4.41 \mathrm{e}-08$ & $4.04 \mathrm{e}-09$ \\
\hline
\end{tabular}

Note: Z-test statistics are in parenthesis; Wald test of constant returns to scale; Proxy variables: raw material expenses. The test for the overidentifying restrictions is based on Sargan-Hansen's J-test.

Note: Industry codes are as follows: 10: Food products; 11: Beverages; 13: Textiles; 14: Wearing apparel; 15: Leather and related products; 16: Wood and products of wood/cork; 17: Paper and paper products; 18: Printing and reproduction of recorded media; 20: Chemicals and chemical products; 21: Pharmaceuticals, medicinal chemicals; 22: Rubber and plastics products; 23: Other non-metallic mineral products; 24: Basic metals; 25: Fabricated metal products; 26: Computer, electronic and optical products; 27: Electrical equipment; 28: Machinery and equipment not elsewhere classified; 29: Motor vehicles, trailers, and semi-trailers; 30: Other transport equipment; 31: Furniture; 33: Repair and installation of machinery and equipment; 34: Other manufacturing.

Source: Authors' estimation from VAES 2010-2016

\subsubsection{Factor elasticities}

We observe capital elasticities ranging from 0.03 to 0.81 . Firms in food products, beverages, chemicals, and chemical products, electrical equipment, motor vehicles, trailers and semi-trailers, and repair and installation of machinery and equipment have the highest capital coefficients, above 0.5 . These sectors are among the smaller sectors in the sample when measured by total value added but yield the highest value-added returns to capital. Firms in wearing apparel have the lowest capital coefficient, below 0.1. Labor elasticities ranging from 0.36 to 1.4. Firms in all sectors, except for fabricated metal products, and repair and installation of machinery and equipment, have labor elasticities above 0.5 . We do not find evidence of constant returns to scale in all sectors, whereas all of them are characterized by increasing returns to scale.

\subsubsection{Some statistics description on TFP}

The descriptive statistics of TFP are presented in Table 2. The best-performing sectors in terms of average productivity in 2010-2016 are wearing apparel (code 14), leather and related products (code 15), group of other manufacturing sectors (code 34), furniture (code 31), computer, electronic and optical products (code 26), and fabricated metal products sectors (code 25). The worst-performing sectors were the production of motor vehicles, trailers and semi-trailers (code 29), beverages (code 11), chemicals and chemical products (code 20), electrical equipment (code 27), and food products (code 10). 
Table 2

Statistic description of TFP by manufacturing industries, 2010-2016

\begin{tabular}{lccc}
\hline Industry & Mean & Min & Max \\
10: Food products & 4.994 & -1.771 & 9.278 \\
11: Beverages & 2.935 & -2.689 & 5.864 \\
13: Textiles & 5.329 & 0.721 & 9.323 \\
14: Wearing apparel & 7.890 & 2.236 & 11.760 \\
15: Leather and related products & 7.853 & 4.165 & 9.941 \\
16: Wood and products of wood/cork & 5.438 & 1.599 & 7.535 \\
17: Paper and paper products & 5.651 & -1.692 & 8.535 \\
18: Printing and reproduction of recorded media & 6.262 & 2.889 & 8.702 \\
20: Chemicals and chemical products & 4.758 & 0.321 & 8.051 \\
21: Pharmaceuticals, medicinal chemicals & 6.050 & 2.486 & 10.166 \\
22: Rubber and plastics products & 5.800 & 0.150 & 8.765 \\
23: Other non-metallic mineral products & 5.304 & -6.892 & 8.463 \\
24: Basic metals & 5.045 & 1.590 & 8.549 \\
25: Fabricated metal products & 6.397 & 1.120 & 9.419 \\
26: Computer, electronic and optical products & 6.407 & 2.670 & 9.921 \\
27: Electrical equipment & 4.827 & 0.981 & 6.734 \\
28: Machinery and equipment not elsewhere classified & 5.501 & 1.196 \\
29: Motor vehicles, trailers and semi-trailers & 2.017 & -1.302 & 3.876 \\
30: Other transport equipment & 5.309 & -0.262 & 8.702 \\
\hline 31: Furniture & 6.901 & 1.572 & 9.853 \\
33: Repair and installation of machinery and equipment & 5.071 & 2.065 & 7.138 \\
34: Other manufacturing & 7.056 & -0.101 & 9.490 \\
\hline All manufacturing & 5.921 & -6.892 & 11.760 \\
\hline
\end{tabular}

Source: Authors' estimation from TFP regressions on VAES 2010-2016

\subsection{Firm heterogeneity and TFP}

\subsubsection{Firm heterogeneity in TFP level}

Column 1 in Table 3 presents average levels of TFP across heterogeneous firm groups for the Vietnamese manufacturing industry. The null hypothesis that TFP levels differ statistically among heterogeneous firm groups is tested using either t-tests or analysis of variance (ANOVA), depending on the number of groups. The ANOVA test is used when firms are divided into three or more groups. It should be noted that the stars on the first, second and third groups and so on from the top represent that the averages between the first and second, first and third, and second and third groups and so on are statistically different in pair-wise tests. The tests show that significant firm heterogeneity in TFP exists for almost all of the firm characteristics considered. Firm size in terms of labor indicates that SME firms have significantly different TFP from big and large ones, big firms are also significantly different from large ones in terms of TFP levels and lower-large firms are also significantly different from upper-big and extreme-large ones in terms of TFP levels. TFP level increases as firm sizes change from a small firm to a medium one, big one and then to a large one. Firm size in terms of capital indicates that SME firms have significantly different TFP from big and large ones, big firms are also significantly different from large ones in terms of TFP levels. However, large firms are not significantly different from extreme-large ones in terms of TFP levels. TFP level decreases as firm sizes change from a small firm to a medium one, big one and then to a large or extreme-large one. With respect to average wage, top-quartile firms paying high wages register much greater TFP than bottom-quartile firms. Foreign ownership shows that FDI firms have significantly higher TFP compared with domestic firms with no foreign ownership (domestic firms). The positive association between foreign ownership and TFP implies that FDI for Vietnamese firms is associated with innovation. In terms of trade, exporting firms have significantly higher TFP compared with non-exporting firms. Among exporters, FDI firms have significantly greater TFP than domestic firms. Regarding imports, importing firms have significantly higher TFP than non-importing firms. Moreover, FDI firms have significantly larger TFP than domestic firms.

\subsubsection{Firm heterogeneity in TFP growth}

Column 2 in Table 3 presents average growth rates in TFP between 2016 and 2010 across heterogeneous firm groups for the Vietnamese manufacturing industry. Firm size in terms of both labor and capital shows that large firms have no significant difference in TFP growth from small firms. Firms paying high wages also have no significant difference in TFP growth from firms paying low wages. Regarding foreign ownership, there is no difference in TFP growth between domestic firms and FDI ones. However, for exporters, FDI firms have significantly higher TFP growth than domestic firms. With regard to imports, there is no difference in TFP growth between domestic non-import firms and FDI ones, and between domestic importing firms and FDI ones as well. 
Table 3

Firm heterogeneity in TFP level for Vietnamese manufacturing firms, 2010-2016

\begin{tabular}{|c|c|c|c|c|c|c|}
\hline \multirow[t]{2}{*}{ Characteristics } & & TFP level & TFP growth & TFP distance & TFP dispersion & FDI-driven TFP spillover \\
\hline & & (1) & (2) & (3) & (4) & (5) \\
\hline \multirow[t]{7}{*}{$\begin{array}{l}\text { Firm size } \\
\text { (labour) }\end{array}$} & Small & $\begin{array}{l}5.672^{\mathrm{a}^{* * *}, \mathrm{~b}^{* * *}, \mathrm{c}^{* * *}} \\
\mathrm{~d}^{* * *}, \mathrm{e}^{* * *}, \mathrm{f}^{* * *} \\
(1.248)\end{array}$ & $1.138^{\mathrm{a}, \mathrm{b}, \mathrm{c}, \mathrm{d}, \mathrm{e}, \mathrm{f}}(1.215)$ & $\begin{array}{l}5.690^{\mathrm{a}^{* * *}, \mathrm{~b}^{* * *}, \mathrm{c}^{* * *}} \\
\mathrm{~d}^{* * *}, \mathrm{e}^{* * *}, \mathrm{f}^{* * *}(1.248)\end{array}$ & $\begin{array}{l}-.003^{\mathrm{a}, \mathrm{b}, \mathrm{c}, \mathrm{d}^{* * *}, \mathrm{e}, \mathrm{f}^{* * *}} \\
(.811)\end{array}$ & $\begin{array}{l}.915^{\mathrm{a} * * *}, \mathrm{~b}^{* * *}, \mathrm{c}^{* * *}, \mathrm{~d}^{* * *}, \mathrm{e}^{* * *}, \\
\mathrm{f}^{* * *}(1.212)\end{array}$ \\
\hline & Lower- medium & $\begin{array}{l}5.896^{b^{* * *}, \mathrm{c}^{* * *}, \mathrm{~d}^{* * *}} \\
\mathrm{e}^{* * * *}, \mathrm{f}^{* * *}(1.473)\end{array}$ & $1.121^{b, c, d, e, f}(2.651)$ & $\begin{array}{l}5.466^{b^{* * *}, \mathrm{c}^{* * *}, \mathrm{~d}^{* * *}} \\
\mathrm{e}^{* * *}, \mathrm{f}^{* * *} \\
(1.473)\end{array}$ & $\begin{array}{l}.021^{\mathrm{b}^{*}, \mathrm{c}^{* * * *}, \mathrm{~d}^{* * *}, \mathrm{e}, \mathrm{f}^{* * *}} \\
(.722)\end{array}$ & $\begin{array}{l}.751^{\mathrm{b}^{* * *}, \mathrm{c}^{* * *}, \mathrm{~d}^{* * *}, \mathrm{e}^{* * *}, \mathrm{f}^{* * *}} \\
(1.380)\end{array}$ \\
\hline & Upper medium & $\begin{array}{l}6.118^{\mathrm{c}^{* * *}, \mathrm{~d}^{* * *}, \mathrm{e}^{* * *},} \\
\mathrm{f}^{* * *}(1.673)\end{array}$ & $1.163^{\mathrm{c}, \mathrm{d}, \mathrm{e}, \mathrm{f}}(.206)$ & $\begin{array}{l}5.243^{\mathrm{c}^{* * *}, \mathrm{~d}^{* * *}, \mathrm{e}^{* * *}} \\
\mathrm{f}^{* * *}(1.673)\end{array}$ & $-.017^{\mathrm{c}, \mathrm{d}, \mathrm{e}, \mathrm{f}^{* * *}}(.699)$ & $\begin{array}{l}.630^{\mathrm{c} * * *}, \mathrm{~d}^{* * *}, \mathrm{e}^{* * *}, \mathrm{f}^{* * *} \\
(1.570)\end{array}$ \\
\hline & Big & $\begin{array}{l}6.239^{\mathrm{d}^{* * *}, \mathrm{e}^{* * *}, \mathrm{e}^{* * *}} \\
(1.807)\end{array}$ & $1.145^{\mathrm{d}, \mathrm{e}, \mathrm{f}}(.579)$ & $\begin{array}{l}5.122^{\mathrm{d}^{* * *}, \mathrm{e}^{* * * *}, \mathrm{f}^{* * *}} \\
(1.807)\end{array}$ & $-.039^{\mathrm{d}, \mathrm{e}, \mathrm{f}^{* * *}}(.694)$ & $.586^{\mathrm{d}^{* * *}, \mathrm{e}^{\mathrm{*} * *}, \mathrm{f}^{* * *}}(1.634)$ \\
\hline & Lower-large & $6.489^{\mathrm{e}^{* * *}, \mathrm{f}^{* * *}}(1.981)$ & $1.170^{\mathrm{e}, \mathrm{f}}(.177)$ & $4.873^{\mathrm{e}^{* * *}, \mathrm{f} * * *}(1.981)$ & $-.053^{\mathrm{e}, \mathrm{f}^{* * *}(.679)}$ & $.526^{\mathrm{e}^{* * *}, \mathrm{f}^{* * *}}(1.884)$ \\
\hline & Upper-large & $7.076^{\mathrm{f***}}(2.259)$ & $1.151^{\mathrm{f}}(.671)$ & $4.286^{\mathrm{f***}}(2.259)$ & $-.013^{\mathrm{f***}}(.702)$ & $.0147^{\mathrm{f**}}(2.237)$ \\
\hline & Extreme-large & $8.769(2.605)$ & $1.188(.222)$ & $2.593(2.605)$ & $.398(.829)$ & $-2.018(2.264)$ \\
\hline \multirow[t]{5}{*}{$\begin{array}{l}\text { Firm size } \\
\text { (capital) }\end{array}$} & Small & $\begin{array}{l}6.125^{\mathrm{a}, \mathrm{b}, \mathrm{c}^{* * *}, \mathrm{~d}^{* * *}} \\
(1.495)\end{array}$ & $1.189^{\mathrm{a}, \mathrm{b}, \mathrm{c}, \mathrm{d}}(.252)$ & $\begin{array}{l}5.237^{\mathrm{a}, \mathrm{b}, \mathrm{c}^{* * *}, \mathrm{~d}^{* * *}} \\
(1.495)\end{array}$ & $\begin{array}{l}-.081^{1^{* * *}, b^{* * *}, c^{* * *}, d^{* * *}} \\
(.771)\end{array}$ & $\begin{array}{l}.5133^{3^{* * *}, \mathrm{~b}^{* * *}, \mathrm{c}^{* * *}, \mathrm{~d}^{* * * *}} \\
(1.445)\end{array}$ \\
\hline & Medium & $\begin{array}{l}6.134^{\mathrm{b}, \mathrm{c}^{* * *}, \mathrm{~d}^{* * * *}} \\
(1.576)\end{array}$ & $1.157^{\mathrm{b}, \mathrm{c}, \mathrm{d}}(1.167)$ & $\begin{array}{l}5.227^{b, c^{* * *}, d^{* * *}} \\
(1.576)\end{array}$ & $-.030^{b^{* * *}, \mathrm{c}^{* * * *}, \mathrm{~d}^{* * *}}(.715)$ & $.652^{\mathrm{b}^{* * *}, \mathrm{c}^{\mathrm{c} * *}, \mathrm{~d}^{* * *}}(1.427)$ \\
\hline & Big & $\begin{array}{l}6.088^{\mathrm{c} * * *, \mathrm{~d}^{* * *}} \\
(1.724)\end{array}$ & $1.099^{\mathrm{c}, \mathrm{d}}(2.829)$ & $\begin{array}{l}5.274^{\mathrm{c}^{* * *}, \mathrm{~d}^{* * *}} \\
(1.724)\end{array}$ & $.032^{\mathrm{c}, \mathrm{d}^{* * *}}(.725)$ & $.7658^{\mathrm{c}^{* * *}, \mathrm{~d}^{* * *}}(1.513)$ \\
\hline & Large & $5.848^{\mathrm{d}}(1.846)$ & $1.119^{\mathrm{d}}(1.221)$ & $5.513^{\mathrm{d}}(1.846)$ & $.0472^{\mathrm{d}^{* * *}}(.742)$ & $1.177^{\mathrm{d}}(1.488)$ \\
\hline & Extreme-large & $5.835(2.010)$ & $1.154(.592)$ & $5.527(2.010)$ & $.128(.784)$ & $1.141(1.650)$ \\
\hline \multirow[t]{4}{*}{ Average wage } & Bottom quartile & $\begin{array}{l}5.441^{\mathrm{a}^{* * *}, \mathrm{~b}^{* * *}, \mathrm{c}^{* * * *}} \\
(1.478)\end{array}$ & $1.070^{\mathrm{a}, \mathrm{b}, \mathrm{c}}(.294)$ & $\begin{array}{l}5.921^{\mathrm{a}^{* * *}, \mathrm{~b}^{* * *}, \mathrm{c}^{* * *}} \\
(1.478)\end{array}$ & $-.578^{\mathrm{a}^{* * *}, \mathrm{~b}^{* * * *}, \mathrm{c}^{* * *}}(.708)$ & $1.206^{a^{* * *}, b^{* * *}, \mathrm{c}^{* * *}}(1.361)$ \\
\hline & Lower quartile & $\begin{array}{l}6.082^{\mathrm{b}^{* * *}, \mathrm{c}^{* * *}} \\
(1.611)\end{array}$ & $1.136^{\mathrm{b}, \mathrm{c}}(.472)$ & $\begin{array}{l}5.280^{\mathrm{b}^{* * * *}, \mathrm{c}^{* * * *}} \\
(1.611)\end{array}$ & $-.121^{\mathrm{b}^{* * *}, \mathrm{c}^{* * *}}(.575)$ & $.666^{\mathrm{b}^{* * *}, \mathrm{c}^{* * *}}(1.423)$ \\
\hline & Upper quartile & $6.385^{\mathrm{c}}(1.749)$ & $1.166^{\mathrm{c}}(.237)$ & $4.976^{\mathrm{c}}(1.749)$ & $.177^{\mathrm{c}^{* * *}}(.553)$ & $.4097^{\mathrm{c}^{* * *}}(1.523)$ \\
\hline & Top quartile & $6.344(1.694)$ & $1.141(2.429)$ & $5.017(1.694)$ & $.522(.628)$ & $.307(1.504)$ \\
\hline \multirow{4}{*}{$\begin{array}{l}\text { Export and } \\
\text { FDI-related } \\
\text { variables }^{\alpha}\end{array}$} & $\begin{array}{l}\text { Domestic non-ex- } \\
\text { ports }\end{array}$ & $\begin{array}{l}5.733^{\mathrm{a}^{* * * *}, \mathrm{~b}^{* * * *}, \mathrm{c}^{* * *}} \\
(1.389)\end{array}$ & $1.116^{\mathrm{a}, \mathrm{b}, \mathrm{c}}(.754)$ & $\begin{array}{l}5.629^{\mathrm{a}^{* * * *}, \mathrm{~b}^{* * * *}, \mathrm{c}^{* * *}} \\
(1.390)\end{array}$ & $-.096^{\mathrm{a}^{* * *}, \mathrm{~b}^{* * * *}, \mathrm{c}^{* * *}}(.733)$ & $.806^{\mathrm{a}^{* * *}}(1.389)$ \\
\hline & FDI non-exports & $\begin{array}{l}6.634^{\mathrm{b}^{* * *}, \mathrm{c}^{* * * *}} \\
(1.902)\end{array}$ & $1.023^{\mathrm{b}, \mathrm{c}}(1.171)$ & $\begin{array}{l}4.727^{\mathrm{b}^{* * * *}, \mathrm{c}^{* * * *}} \\
(1.902)\end{array}$ & $.335^{\mathrm{b}^{* * *}, \mathrm{c}^{* * *}}(.779)$ & \\
\hline & Domestic exports & $5.974^{\mathrm{c}^{* * *}}(1.634)$ & $1.112^{\mathrm{c}}(1.240)$ & $5.388^{\mathrm{c}^{* * *}}(1.634)$ & $-.068^{c^{* * *}}(.656)$ & $.565(1.634)$ \\
\hline & FDI exports & $6.511(1.919)$ & $1.132(2.419)$ & $4.851(1.919)$ & $.117(.761)$ & \\
\hline \multirow{4}{*}{$\begin{array}{l}\text { Import and } \\
\text { FDI-related cat- } \\
\text { egories }^{\alpha}\end{array}$} & $\begin{array}{l}\text { Domestic non-im- } \\
\text { ports }\end{array}$ & $\begin{array}{l}5.769^{\mathrm{a}^{* * * *}, \mathrm{~b}^{\mathrm{*} * *}, \mathrm{c}^{* * *}} \\
(1.410)\end{array}$ & $1.101^{\mathrm{a}, \mathrm{b}, \mathrm{c}}(1.230)$ & $\begin{array}{l}5.593^{\mathrm{a}^{* * *}, \mathrm{~b}^{* * *}, \mathrm{c}^{* * *}} \\
(1.410)\end{array}$ & $-.088^{\mathrm{a}^{* * * *}, \mathrm{~b}^{* * * *}, \mathrm{c}^{* * * *}}(.724)$ & $.770^{\mathrm{a}^{* * *}}(1.410)$ \\
\hline & FDI non-imports & $\begin{array}{l}6.762^{\mathrm{b}^{* * *}, \mathrm{c}^{* * *}} \\
(1.870)\end{array}$ & $1.131^{\mathrm{b}, \mathrm{c}}(.834)$ & $\begin{array}{l}4.599^{\mathrm{b}^{* * * *}, \mathrm{c}^{\mathrm{*} * *}} \\
(1.870)\end{array}$ & $.291^{\mathrm{b}^{* * *}, \mathrm{c}^{* * *}}(.756)$ & \\
\hline & Domestic imports & $5.955^{\mathrm{c}^{* * * *}}(1.667)$ & $1.136^{\mathrm{c}}(.340)$ & $5.407^{\mathrm{c}^{* * *}}(1.667)$ & $-.080^{c^{* * *}}(.658)$ & $.585(1.667)$ \\
\hline & FDI imports & $6.448(1.927)$ & $1.125(2.527)$ & $4.914(1.927)$ & $.117(.771)$ & \\
\hline
\end{tabular}

Note: $\alpha$ : The period is 2010-2015. Standard error in the parentheses.

$* * *, * *, *$ : The averages are statistically different at the $1 \%, 5 \%, 10 \%$ level.

For multiple groups, the stars on $1^{\text {st }}, 2^{\text {nd }}$ and $3^{\text {rd }}$ and so on groups from the top represent the averages between $1^{\text {st }}$ and $2^{\text {nd }}$, $1^{\text {st }}$ and $3^{\text {rd }}$, and $1^{\text {st }}$ and $4^{\text {th }}$ groups and so on are statistically different, respectively. For example, a, b, c, d, e, f in column (1) and for firm size in terms of labor denotes the difference between small firms and lower-medium firms, upper-medium firms, big firms, lower-large firms, upper-large firms, and extreme-large firms, respectively.

The symbols a, b, c, d, e in column (1) and for firm size in terms of capital denotes the difference between small firms and medium firms, big firms, large firms, and extreme-large firms, respectively. The symbols a, b, c, d in column (1) and for firm's average wage denote the difference between the firms in the bottom quartile, the lower quartile, the upper quartile, and the top quartile, respectively.

Source: Authors' estimation from VAES 2010-2016

\subsubsection{Firm heterogeneity in TFP distance}

Column 3 in Table 3 presents TFP distance across heterogeneous firm groups for the Vietnamese manufacturing industry. The null hypothesis that TFP distance differ statistically among heterogeneous firm groups is tested using analysis of variance (ANOVA). The tests show that significant firm heterogeneity in TFP distance exists for almost all of the firm characteristics considered. Firm size in terms of labor indicates that SME firms have significantly different TFP distance from big and large ones, big firms are also significantly different from large ones in terms of TFP distance and lower-large firms are also significantly different from upper-big and extreme-large ones in terms of TFP distance. TFP distance decreases as firm sizes change from a small firm to a medium one, big one and then to a large one. Firm size in terms of capital indicates that SME firms have significantly different TFP distance from big and large ones, big firms are also significantly different from large ones in terms of TFP distance. TFP distance increases as firm sizes change from a small firm to a medium one, big one and then to a large or extreme one. With respect to average wage, top-quartile firms paying high wages register much lower TFP distance than bottom-quartile firms. Foreign ownership shows that FDI firms have significantly lower TFP distance compared with domestic firms with no foreign ownership (domestic firms). The negative association between foreign ownership and TFP distance implies that FDI for Vietnamese firms is associated with innovation and catch-up. In terms of trade, exporting firms have significantly lower TFP distance compared with non-exporting firms. Among exporters, FDI firms have significantly lower TFP distance than domestic firms. With reference to imports, importing firms have significantly lower TFP distance than non-importing firms. Moreover, FDI firms have significantly smaller TFP distance than domestic firms.

\subsubsection{Firm heterogeneity in TFP dispersion}

Column 4 in Table 3 presents average levels of TFP dispersion across heterogeneous firm groups for the Vietnamese manufacturing industry. The null hypothesis that TFP dispersion differs statistically among heterogeneous firm groups is tested 
using analysis of variance (ANOVA). Firm size in terms of labor indicates that SME firms have significantly different TFP dispersion from large ones, big firms are also significantly different from large ones in terms of TFP dispersion and lowerlarge firms are also significantly different from extreme-large ones in terms of TFP dispersion. TFP dispersion increases as firm sizes change from a small firm to a medium one, big one and then to a large one. Firm size in terms of capital indicates that SME firms have significantly different TFP dispersion from big and large ones, big firms are also significantly different from large ones in terms of TFP dispersion. TFP dispersion decreases as firm sizes change from a small firm to a medium one, big one and then to a large or extreme-large one. With respect to average wage, top-quartile firms paying high wages register much higher TFP dispersion than bottom-quartile firms. Foreign ownership shows that FDI firms have significantly higher TFP dispersion compared with domestic firms with no foreign ownership (domestic firms). In terms of trade, exporting firms have significantly higher TFP dispersion compared with non-exporting firms. Among exporters, FDI firms have significantly higher TFP dispersion than domestic firms. In relation to imports, importing firms have significantly higher TFP dispersion than non-importing firms. Moreover, FDI firms have significantly larger TFP dispersion than domestic firms.

\subsubsection{Firm heterogeneity in FDI-driven TFP spillover effects}

Column 5 in Table 3 presents average levels of FDI-driven TFP spillover effects across heterogeneous firm groups for the Vietnamese manufacturing industry. The null hypothesis that FDI-driven TFP spillover effects differ statistically among heterogeneous firm groups is tested using analysis of variance (ANOVA). Firm size in terms of labor indicates that SME firms have significantly different FDI-driven TFP spillover effects from big and large ones, big firms are also significantly different from large ones in terms of FDI-driven TFP spillover effects and lower-large firms are also significantly different from upper and extreme-large ones in terms of FDI-driven TFP spillover effects. FDI-driven TFP spillover effects decrease as firm sizes change from a small firm to a medium one, big one and then to a large one. Firm size in terms of capital indicates that SME firms have significantly different FDI-driven TFP spillover effects from big and large ones, big firms are also significantly different from large ones in terms of FDI-driven TFP spillover effects. FDI-driven TFP spillover effects increase as firm sizes change from a small firm to a medium one, big firm and then to a large or extreme-large one. With respect to average wage, top-quartile firms paying high wages register much lower FDI-driven TFP spillover effects than bottom-quartile firms. In terms of trade, exporting firms have significantly lower FDI-driven TFP spillover effects compared with non-exporting firms. With regard to imports, importing firms have significantly lower FDI-driven TFP spillover effects than non-importing firms.

\section{Conclusion and implications}

This paper has examined the heterogeneity and productivity performance using a sample of firms from the Vietnamese manufacturing industries for the period 2010-2016. To this end, firm productivities were estimated with the Ackerberg et al. (2015), using the deflated gross value added (LY) of firms as a measure of output, and the intermediate inputs (raw material) as a proxy to avoid the bias problem. Firm heterogeneity is analyzed not only along with firms' structural features such as size in terms of labor, capital, average wage rate, but also in line with their business characteristics such as trade (export and import) and FDI. Firm heterogeneity with regard to decomposed TFP components has shown significant firm heterogeneity in many decomposed productivity components of the Vietnamese manufacturing industry. Firm heterogeneity in TFP and trade also has shown that there was a clear gap in TFP, favoring exporters over non-exporters. Likewise, there was a clear gap in TFP favoring importers over non-importers. This suggests that the beneficial impact of exports or imports on productivity was realized most when firms become exporters or importers. With respect to TFP growth, unlike the TFP level, the patterns of trade heterogeneity were not clearly proved. In addition, exporting (and/or importing) firms have had significantly lower TFP distance compared with non-exporting (and/or non-importing) firms. Similarly, exporting (and/or importing) firms have maintained significantly higher TFP dispersion compared with non-exporting (and/or non-importing) firms. Moreover, exporting (and/or importing) firms have yielded significantly lower FDI-driven TFP spillover effects compared with non-exporting (and/or non-importing) firms. Regarding foreign ownership, there was no difference in TFP growth between domestic firms and FDI ones. However, for exporters, FDI firms have had significantly higher TFP growth than domestic firms. With regard to imports, there is no difference in TFP growth between domestic non-import firms and FDI ones, and between domestic importing firms and FDI ones as well. Regarding TFP distance, among exporters, FDI firms have significantly lower TFP distance than domestic firms. Moreover, FDI firms have significantly smaller TFP distance than domestic firms. With respect to TFP dispersion, FDI firms have significantly larger TFP dispersion than domestic firms.

The study also has shown that firm heterogeneity in productivity sources was much more complex than the heterogeneity in productivity as a whole. Firm heterogeneity in productivity sources delivers more information about firm-specific differences in productivity. For example, in terms of labor, the larger firms are, the more productive they are. In terms of capital stocks, TFP level decreases as firm sizes change from a small firm to a medium one, big one and then to a large or extreme-large one. With respect to average wage, top-quartile firms paying high wages register much greater TFP than bottom-quartile firms. With respect to TFP growth, firm size in terms of both labor and capital shows that large firms have no significant difference in TFP growth from small firms. Firms paying high wages also have no significant difference in TFP growth from firms paying low wages. Regarding TFP distance, TFP distance decreases as firm sizes in terms of labor change from a small firm to a medium one, big one and then to a large one, whereas TFP distance increases as firm sizes in terms of capital stocks change from a small firm to a medium one, big one and then to a large or extreme-large one. With respect to average wage, 
top-quartile firms paying high wages register much lower TFP distance than bottom-quartile firms. In regard to TFP dispersion, TFP dispersion increases as firm sizes in terms of labor change from a small firm to a medium one, big one and then to a large one, whereas TFP dispersion decreases as firm sizes in terms of capital stocks change from a small firm to a medium one, big one and then to a large or extreme-large one. With respect to average wage, top-quartile firms paying high wages register much higher TFP dispersion than bottom-quartile firms. Last but not least, regarding FDI-driven TFP spillover effects, FDI-driven TFP spillover effects decrease as firm sizes in terms of labor change from a small firm to a medium one, big one and then to a large one. In addition, FDI-driven TFP spillover effects increase as firm sizes in terms of capital stocks change from a small firm to a medium one, big firm and then to a large or extreme-large one. With respect to average wage, topquartile firms paying high wages register much lower FDI-driven TFP spillover effects than bottom-quartile firms. In conclusion, heterogeneity of firm effects on TFP certainly provoke policies expanding SMEs in terms of capital stocks and obtaining advantages of labor costs, paying higher wage rate, involving into the trade, and may having the foreign capital investment.

\section{Acknowledgement}

This research is funded by Vietnam National University - Ho Chi Minh City under grant number C2018-34-05.

\section{References}

Ackerberg, D., Caves, K., \& Frazer, G. (2006). Structural estimation of production functions. manuscript. Department of Economics, UCLA.

Ackerberg, D. A., Caves, K., \& Frazer, G. (2015). Identification properties of recent production function estimators. Econometrica, 83(6), 2411-2451.

Bernard, S., Wirth, R., Schreiber, A., Schulz, H.-M., \& Horsfield, B. (2012). Formation of nanoporous pyrobitumen residues during maturation of the Barnett Shale (Fort Worth Basin). International Journal of Coal Geology, 103, 3-11.

Bloom, N., Draca, M., \& Van Reenen, J. (2016). Trade induced technical change? The impact of Chinese imports on innovation, IT and productivity. The review of economic studies, 83(1), 87-117.

Eaton, J., Kortum, S., \& Kramarz, F. (2011). An anatomy of international trade: Evidence from French firms. Econometrica, 79(5), 1453-1498.

Griliches, Z., \& Mairesse, J. (1995). Production Functions: The Search for Identification. Retrieved from https://EconPapers.repec.org/RePEc:nbr:nberwo:5067

Kasahara, H., \& Lapham, B. (2013). Productivity and the decision to import and export: Theory and evidence. Journal of International Economics, 89(2), 297-316.

Levinsohn, J., \& Petrin, A. (2003). Estimating production functions using inputs to control for unobservables. The review of economic studies, 70(2), 317-341.

Melitz, M. (2003). The impact of trade on aggregate industry productivity and intra-industry reallocations. Econometrica, 71(6), 1695-1725.

Ngo, Q.-T., \& Nguyen, C. T. (2019). Do export transitions differently affect firm productivity? Evidence across Vietnamese manufacturing sectors. Post-Communist Economies, 1-27.

Nguyen, H., Le, D., Dang, D., \& Nguyen, T. (2020). Labor heterogeneity and total factor productivity: evidence from Vietnamese manufacturing private sector. Management Science Letters, 10(1), 29-40.

Nguyen, H. Q. (2017). Business reforms and total factor productivity in Vietnamese manufacturing. Journal of Asian Economics, 51, 33-42.

Olley, G. S., \& Pakes, A. (1996). The Dynamics of Productivity in the Telecommunications Equipment Industry. Econometrica, 64(6), 1263-1298.

Petrin, A., \& Levinsohn, J. (2012). Measuring aggregate productivity growth using plant level data. The Rand journal of economics, 43(4), 705-725.

Syverson, C. (2011). What determines productivity? Journal of Economic literature, 49(2), 326-365.

Wooldridge, J. M. (2009). On estimating firm-level production functions using proxy variables to control for unobservables. Economics Letters, 104(3), 112-114.

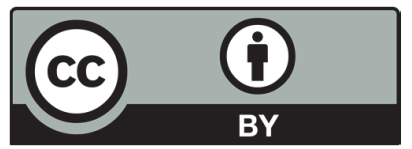

(C) 2020 by the authors; licensee Growing Science, Canada. This is an open access article distributed under the terms and conditions of the Creative Commons Attribution (CC-BY) license (http://creativecommons.org/licenses/by/4.0/). 